\title{
Comparison of Admittance Plots for Glass Carbon (GC) and Edge Plane Pyrolytie Graphite (EPPG) Electrodes in Three
}

\section{Typical Kinds of Electrolytes}

\author{
K. Ding ${ }^{\mathrm{a}, \mathrm{b}} *$ and Q. Wang $^{\mathrm{a}}$ \\ a Chemistry College, Hebei Teacher's University, Shijiazhuang 050016, P. R. China \\ ${ }^{\mathrm{b}}$ Department of Electronic Chemistry, Interdisciplinary Graduate School of Science and \\ Engineering, Tokyo Institute of Technology, Midori-ku, Yokohama 226-8502, Japan
}

Received $1^{\text {st }}$ September 2006; accepted $8^{\text {th }}$ November 2006

\begin{abstract}
Electrochemical response of glass carbon (GC) and edge plane pyrolytic graphite (EPPG) electrodes was investigated using electrochemical impedance spectroscopy (EIS) method in three kinds of electrolytes, i.e., $0.1 \mathrm{M} \mathrm{Na}_{2} \mathrm{SO}_{4}$ (aqueous solution), 0.1 $\mathrm{M}$ tetraethylammonium perchlorate (TEAP) (acetonitrile solution) and pure room temperature ionic liquids (RTILs) of 1-ethyl-3-methylimidazolium tetrafluorobroate (EMIBF4). The marked difference in the admittance plots was exhibited by the GC electrode, suggesting that the double layer occurring on GC electrode differed from each other when immersed in different electrolytes, indirectly proving the mesoporosity structure of GC electrode. While for EPPG electrode, similar results were obtained in these three selected electrolytes, which were interpreted by the "defects" in EPPG electrode.
\end{abstract}

Keywords: glass carbon electrode, edge plane pyrolytic graph (EPPG) electrode, room temperature ionic liquids (RTILs), admittance plot.

\section{Introduction}

The aim of this paper is to report the electrochemical behavior of glass carbon (GC) and EPPC electrodes in three typical electrolyte solutions commonly employed in electrochemistry, i.e., aqueous solution, organic solution and ionic liquids, with the intention to reveal the influence of the electrolyte on the interface between electrolyte and electrode.

Due to its excellent characteristics such as hardness and shape stability compared to other carbon material, GC material has received considerable attentions in the fields of battery and semiconductor industries [1,2]. Features of GC closely relate

\footnotetext{
* Corresponding author. E-mail address: dkeqiang@263.net
} 
with their preparation process, hence, many works concerning their preparation technique were carried out with an intention to obtain satisfactory GC materials. Meanwhile, the well-defined conductivity allowed GC electrodes to be widely applied in electrochemistry. Comparing with other kinds of electrodes, due to their porous structure or to their suspended valence, being able to be "tailored" with different kinds of organic groups was thought to be alluring point of GC electrode $[3,4]$.

Edge plane pyrolytic graphite (EPPG) electrode is one kind of pyrolytic graphite. As reported, pyrolytic graphite electrode usually was divided as the basal plane pyrolytic graphite (BPPG) electrode, containing graphite layers parallel to the working surface [5]. Also, generally speaking, edge plane pyrolytic graphite (EPPG) electrodes are advantageous compared to BPPG and GC electrodes, since the highly reactive edge plane "defects" of EPPG electrode allowed low detection limits, high sensitivities, improved signal to noise characteristics and low over-potentials [6-8], which promoted us to detect EPPG electrode.

Room temperature ionic liquids (RTILs) are the novel media between water and organic phase; despite their many advantages presented by numerous papers, their most charming application in electrochemistry was that due to their higher conductivity; compared to the ordinary organic solvent, RTILs could be utilized as both solvent and electrolyte, that is to say, RTILs were employed as "green electrolytes" where no solvent was involved [9]. As stated previously, due to RTILs' particular properties, the concept of double layer originated from RTILs still kept ambiguous; for example, Graves and Inman [10] have developed two peculiar models to interpret double layer constructed in RTILs; in the first model, it was suggested that for a given charge density on the metal electrode there is an equal and opposite charge density on the melt produced by anion and cation vacancies; unfortunately, this model failed to predict the double layer capacitance values for those whose anionic and cationic radii were not equal. The second model was denoted as multiplayer model, the anions and cations in the molten salt were thought to produce an ordered melt structure comprised of several layers of anions and cations at the interface, but, failing to predict the double layer capacitance as a function of temperature [10]. In addition, the literature data on the capacitance of the electrode/RTILs interface are limited to a few studies [11]. The ambiguous conclusion about double layer capacitance established in RTILs intrigued us to continue the measurement of the capacitance value in RTILs.

With the emergence of RTILs, the electrolyte, used in electrochemistry, has grouped as three typical kinds, i.e., aqueous electrolytes containing inorganic salts dissolved in water, organic electrolytes having quaternary ammonium salt solved in common organic solvent, and the last novel generated electrolyte of RTILs where solvent was not utilized. Therefore, in the following work, three typical electrolytes were selected, i.e., 0.1 $\mathrm{M} \mathrm{Na}_{2} \mathrm{SO}_{4}$ (aqueous solution), $0.1 \mathrm{M}$ tetraethylammonium perchlorate (denoted as TEAP) / acetonitrile solution, and pure room temperature ionic liquids (RTILs) of 1-ethyl-3-methylimidazolium tetrafluorobroate $\left(\mathrm{EMIBF}_{4}\right)$. 
To the best of our knowledge, no paper reporting the comparison of the electrochemical response in the above three kinds of electrolytes was published; probably, it was too simple to be considered by other groups. But, in our case, some interesting results were obtained. For example, GC electrode exhibited different response in the admittance plots recorded in EIS, at least indicating that the double layer occurring on GC electrode was closely related to the solvent used, though the exact surface configuration of GC electrode could not be achieved by the present method. While for EPPG electrode, similar results were attained in these three kinds of electrolyte. Basing on our results, combining with previous reports, possible interface structure of GC electrode was proposed, which is helpful not only for the all-around comprehension of GC and EPPG electrodes, but also for the further investigation of RTILs.

\section{Results and discussion}

\section{Electrochemical response of GC electrode in three kinds of electrolyte}

Cyclic voltammetry was firstly used to detect the electrochemical response of GC electrode in the selected three electrolytes. Unfortunately, no helpful information was drawn out since the value of capacitance correlates with many factors, for instance, the dielectric constant of electrolyte, the radii of molecules used to construct the double layer, etc.

EIS is another powerful technique to describe the surface configuration of the electrode. Among these obtained plots, Nyquist plots were the most frequently used figures to indicate the electrochemistry response; in our case, Nyquist plots were also obtained, where only lines nearly perpendicular to the real resistance axis were obtained, from which the information about the double layer was hardly acquired [12]. So the admittance plots for GC were presented in Fig. 1, where the evident distinction exhibited in three kinds of electrolytes was showed definitely. As shown in Fig. 1, in the common aqueous solution of $0.1 \mathrm{M}$ $\mathrm{Na}_{2} \mathrm{SO}_{4}$, a well-defined semicircle was presented, hinting an ideal double layer, which was represented by a circuit consisting of a capacitor element in series with the solution resistant element, constructed on GC electrode surface. While in the case of $0.1 \mathrm{M}$ TEAP, the positive value of admittance was showed in the forth quadrant, suggesting that, except for the capacitor behavior at the lower frequency region, the inductor element was expressed in the higher frequency region [13], for the case of RTILs of $\mathrm{EMIBF}_{4}$ apart from the capacitor behavior exhibited at lower frequency region, a line nearly parallel to the image part of admittance was observed in the higher frequency, implying that in the higher frequency region, the imitated circuit consisted of a resistance element parallel to a capacitor element [13]. The differed figures shown in Fig. 1 at least indicated that the double layer constructed on GC electrode in above three kinds of solution was different from each other, though the exact distinction of these double layers could not be obtained by $\mathrm{CV}$ and EIS methods. 


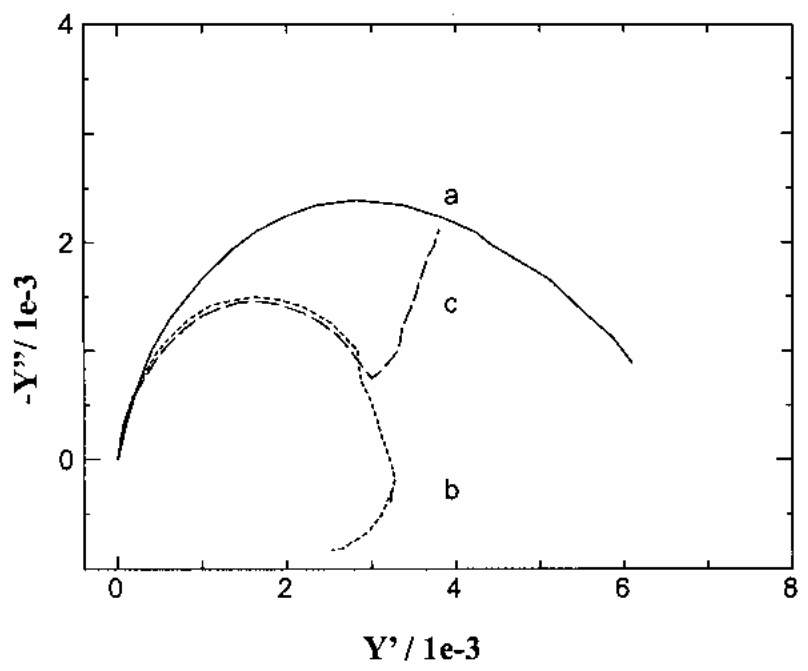

Figure 1. Admittance plots of $\mathrm{GC}$ electrode in different electrolytes, a: in $0.1 \mathrm{M}$ $\mathrm{Na}_{2} \mathrm{SO}_{4}$, b: in $0.1 \mathrm{M}$ TEAP, c: in $\mathrm{EMIBF}_{4}$, which was measured at the open circuit potential.

The relationship between the applied frequency and the varied phase angle was also clearly displayed in Fig. 2, where the main difference was seen in the higher frequency region, i.e., $10^{4}$ to $10^{5} \mathrm{~Hz}$; for example, for the case of aqueous electrolyte shown by line a, a line parallel to the phase angle axis was exhibited, while for the case of organic electrolyte exhibited by line $b$, a line with negative slope was presented; interestingly, for the case of RTILs of EMIBF 4 (as shown by line c), a line with positive slope was seen at the same frequency region. Meanwhile, at the lower frequency region, line $b$ exhibited a line perpendicular to the phase axis in the shorter frequency region from 1 to about $60 \mathrm{~Hz}$, line a and line $\mathrm{c}$ have the similar behavior from 1 to $10^{2} \mathrm{~Hz}$. Fig. 2 substantially supported that GC electrodes presented varied behavior in different electrolytes. That is to say, the surface configuration of GC electrode was altered by the introduction of different electrolytes. How to understand this interesting result?

As reported by Nishikarna's group [14], the pores in carbon materials played an important role in carbon's function and characteristics, and the pores in GC are closed for air and moisture. According to this proposition, we think, in the aqueous electrolyte, due to the c pores, GC electrode only exhibits like a mirror surface, on which an ideal capacitor could be established, consistent with the results shown by Fig. 1 and Fig. 2. While in the organic electrolyte, pores on GC opened in some degree, subsequently, electrolyte molecules could insert into the pores, behaving like an inductor. When immersed in RTILs of EMIBF 4 , owing to the lowered polarity of RTILs, pores in GC were entirely opened, giving rise to the circuit composed by resistance element parallel to a capacitor element in the higher frequency region. 


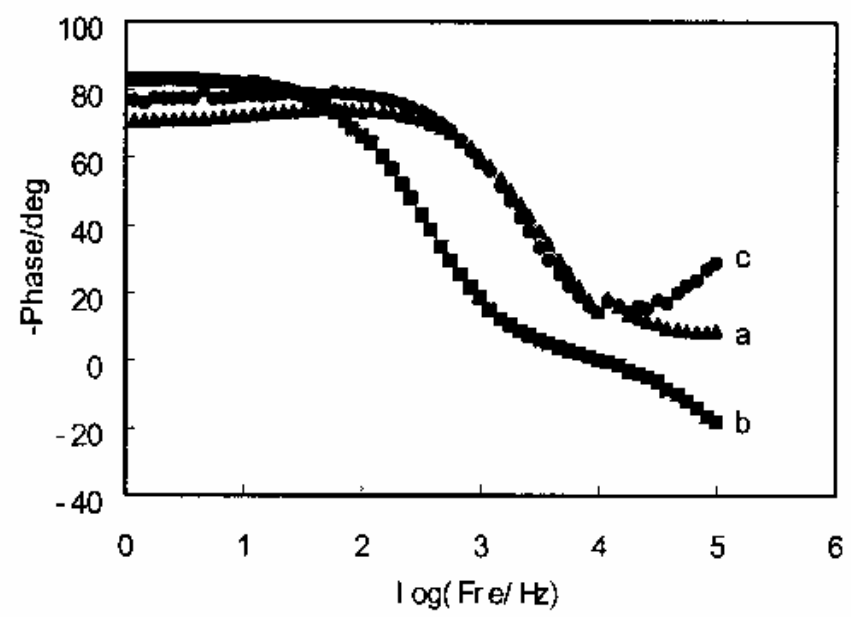

Figure 2. Relationship between frequency and phase angle for GC electrode. a: in 0.1 $\mathrm{M} \mathrm{Na}_{2} \mathrm{SO}_{4}$, b: in $0.1 \mathrm{M}$ TEAP, c: in $\mathrm{EMIBF}_{4}$.

\section{Electrochemical behavior of EPPG electrode in three kinds of electrolytes}

Interestingly, as shown by Fig. 3, except for the varied-diameter semicircles, the similar admittance plots were obtained for EPPG electrode in the selected three kinds of electrolytes, hinting that the surface configuration of EPPG electrode was not altered by the introduction of varied electrolytes. The similar behavior was also clearly reflected by the relationship between frequency and phase angle illustrated by Fig. 4.

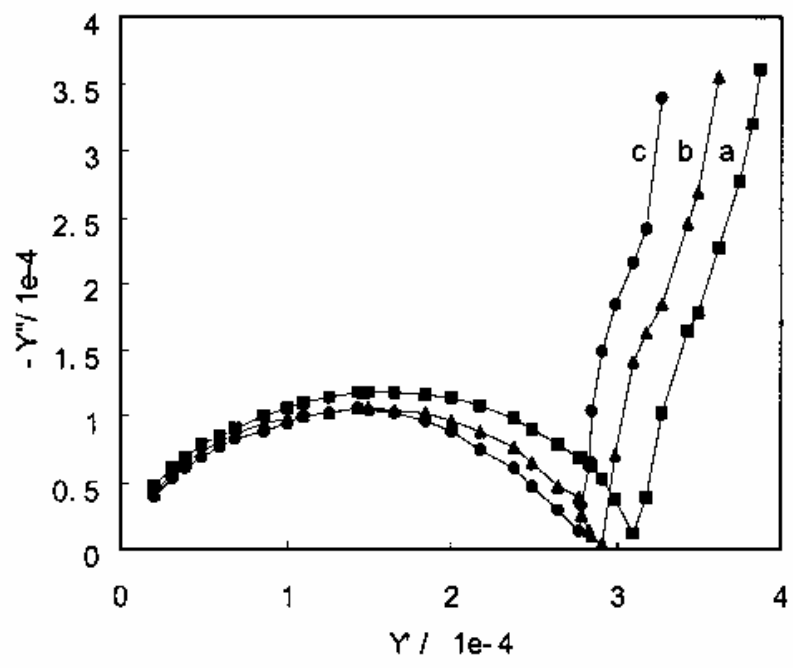

Figure 3. Admittance plots of GC electrode. a: in $0.1 \mathrm{M} \mathrm{Na}_{2} \mathrm{SO}_{4}$, b: in $0.1 \mathrm{M}$ TEAP, c: in $\mathrm{EMIBF}_{4}$.

EPPG electrode has been well described by Compton [6,7], where the significant catalysis exhibited by EPPG was attributed to the "defects" in the layer step of EPPG electrode. Therefore, we think the behavior expressed in the higher frequency region, for example, the line with positive slope shown in the 
frequency range from $10^{4}$ to $10^{5} \mathrm{~Hz}$ in Fig. 3, were all resulted from the "defects" existing in EPPG electrode.

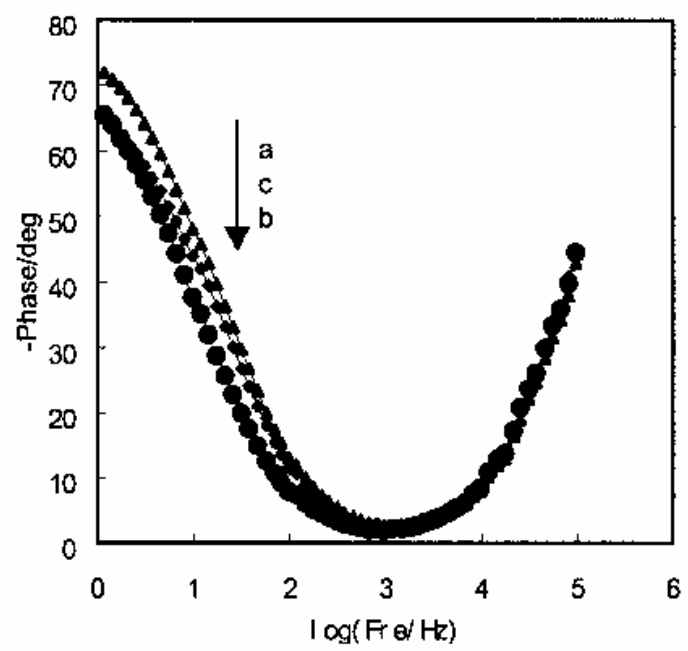

Figure 4. Relationship between frequency and phase angle for $\mathrm{GC}$ electrode. a: in 0.1 $\mathrm{M} \mathrm{Na}_{2} \mathrm{SO}_{4}$, b: in 0.1 M TEAP, c: in $\mathrm{EMIBF}_{4}$.

Hence, according to our results and the former published proposition, we think the surface configuration of GC electrode undergone a variation when dipped in different electrolytes, while EPPG electrode exhibited the identical surface configuration when immersed in different electrolytes.

The following scheme probably can describe the surface configuration change of GC electrode very well.

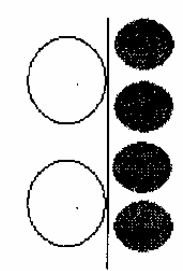

Aqueous electrolyte

Organic electrolyte

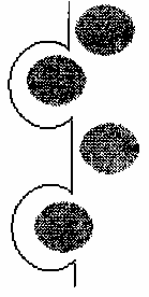

$\mathrm{EMIBF}_{4}$

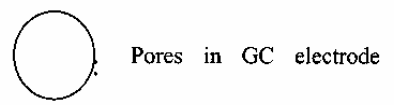

Molecules aligned on the surface of GC electrode

Scheme 1. Surface scheme of GC electrode in three typical kinds of electrolytes.

Scheme 1 clearly describes the state of pores in GC electrode when immersed in different kinds of electrolyte. In other words, pores of GC electrode will adjust themselves when connecting with different electrolytes. 


\section{Final comments}

In this work, electrochemical behavior of GC and EPPG electrodes was compared in three typical kinds of electrolyte solutions; the obtained results suggested that GC electrode displayed varied surface configuration when introduced into different electrolytes, according with the previous proposition very well. While for EPPG electrode, similar behaviors were observed in three electrolytes, which were interpreted by the "defects" in EPPG electrode. We must admit CVs and EIS were techniques reflecting the macro response generated by the electrochemical process, the exact micro configuration variation of electrodes should be detected by other more direct techniques. Presenting the varied admittance plot of GC electrode in three typical electrolytes, and comparing the admittance plots of GC with that of EPPG electrode in RTILs is the main contribution of this preliminary work.

\section{References}

1. S. Yamada, H. Sato, Nature 193 (1962) 261.

2. M. Pérez-Mendoza, C. Schumacher, E. Suárez-Garcia, M.C. AlmazánAlmazán, M. Domingo-García, E.J. Lópe-Gazrzón, N.A. Seaton, Carbon 44 (2006) 638.

3. Y. Korai, K. Sakamoto, I. Mochida, O. Hirai, Carbon 42 (2004) 219.

4. X. Wang, G.M. Zhang, Y.L. Zhang, F.Y. Li, R.C. Yu, C.Q. Jin, G.T. Zou, Carbon 41 (2003) 179.

5. C.E. Banks, T.J. Davies, G.G. Wildgoose, R.G. Compton, Chem. Commun. 17 (2005) 829.

6. C.E. Banks, R.G. Compton, Anal. Sci. 21 (2005) 1263.

7. C.E. Banks, R.R. Moore, T.J. Davies, R.G. Compton, Chem. Commun. 16 (2004) 1804.

8. C.E. Banks, A. Crossley, C. Salter, S.J. Wilkins, R.G. Compton, Angew. Chem. Int. Ed. 45 (2006) 2533.

9. T. Welton, Chem. Rev. 99 (1999) 2071.

10. A.D. Graves, D. Inman, Chem. Rev. 25 (1970) 357.

11. C. Nanjundiah, S.F. McDevitt, V.R. Koch, J. Electrochem. Soc. 144 (1997) 3392.

12. K.Q. Ding, Q. Wang, Z. Jia, R. Tong, X. Wang, H. Shao, J. Chin. Chem. Soc. 50 (2003) 387.

13. C.N. Cao, J.Q. Zheng, An introduction to electrochemical impedance spectroscopy, Science Publishing Company, 2002, page: from 7 to 15.

14. K. Fukuyama, T. Nishizawa, K Nishikawa, Carbon 39 (2001) 2017. 